Method. An audit of patients MDT medical notes on 38 admitted to Angelton clinic was carrired out in March. It was documented if the patient had a clear DNAR or Escalation plan that was easily accessible in the front of the notes. The guidelines compared to were the GMC recommendations that patients 12 months of should have a discussion about risks and benefits associated with Cardiopulmonary Resuscitation. If the patient lacks capacity a best interest decision should be made with nearest relatives. Discussions should also be had with patients and family in in regards to and transfer to a medical ward.

Upon completion of the initial PDSA cycle, views were sought from the wider MDT a new escalation of care proforma was designed. This was implemented by education and communication with members of the medical team. This was to be clearly placed in the notes, with the DNAR form if that was appropriate. Result. All inpatient notes were audited at Angelton Clinic in March 2020. It was found that only $18 \%$ of patients had Escalation of Care plans in comparison to $84 \%$ of notes which had DNAR forms. Previous escalation of care forms were not being utilised appropriately.

Upon implementation of the Escalation of Care proforma, a re-audit of the audit cyle was completed. In July 2020 it was found that $78 \%$ of notes had completed Escalation of Care forms with $83 \%$ had completed DNAR forms.

Conclusion. To enable ongoing sustained improvement, the unit Nurse Practitioner will champion its completion. The audit findings have been shared with the newly rotated junior doctors and proformas were made available on all inpatient wards.

You have to acknowledge the problem before you can address the problem: Audit looking at identification of co-existing substance misuse in a Liaison Psychiatry patient population

Emma McLean ${ }^{1 \star}$, Akash Kadiwar ${ }^{2}$ and Mariam Alexander ${ }^{3}$

${ }^{1}$ Imperial College Healthcare NHS Trust; ${ }^{2}$ Northampton General Hospital NHS Trust and ${ }^{3}$ West London NHS Trust

${ }^{\star}$ Corresponding author.

doi: 10.1192/bjo.2021.276

Aims. To evaluate if patients referred to Ealing Liaison Psychiatry Service (ELPS) with co-existing substance use are being appropriately identified as per NICE guidelines.

Patients with co-existing substance misuse have greater morbidity and mortality and it is therefore important to identify these patients to optimise their management. NICE recommends that all patients are asked about their substance use.

Anecdotally, our team felt we were doing a good job of identifying and managing such patients but we had no objective evidence of this.

Method. Completed a retrospective audit looking at a sample of patients referred to ELPS over two weeks in December 2019.

A training session for ELPS was then held to highlight the initial audit results and NICE guideline recommendations.

We then repeated the audit over two weeks in March 2020.

Result. Initial audit (100 patients):

Only $69 \%$ of patients asked about substance use. From those asked, 50-65.2\% were using a substance, most commonly alcohol.

None of the patients over the age of 80 were asked about substance use vs $79.5 \%$ of patients aged $20-40$ years.

$55 \%$ of females vs $81 \%$ of males were asked about illicit substances.

$33.3 \%$ of ward referrals vs $74.2 \%$ of Emergency Department referrals asked about substance use
Re-audit (53 patients):

Significant improvement across all areas

93\% now asked about substance use

$60 \%$ of over 80 s, $96 \%$ of females and $85 \%$ of ward referrals were now being correctly asked about substance use

Conclusion. We were surprised to find that we were initially not meeting NICE standards regarding asking patients about their substance use.

Acknowledging this problem during our training session proved to be effective.

This knowledge will help us develop our care pathways with our Acute colleagues and the Drug and Alcohol Liaison Service.

\section{Antipsychotic prescribing in dementia}

James McLoughlin ${ }^{1 *}$, Emma Roemmele ${ }^{2}$, Marguerite Cryan ${ }^{2}$, Catherine Dolan ${ }^{2}$ and Geraldine McCarthy ${ }^{2}$

${ }^{1}$ Psychiatry Department, University Hospital Galway and ${ }^{2}$ Sligo

University Hospital

${ }^{\star}$ Corresponding author.

doi: 10.1192/bjo.2021.277

Aims. The majority of people with dementia will develop one or more behavioural or psychological symptoms of dementia (BPSD) as the illness progresses. Treating these symptoms in diverse residential environments is a challenge, with frequent prescribing of antipsychotic medications. The risks and limited benefits of antipsychotic use in this context are well recognised, prompting national guidelines in Ireland to improve prescribing patterns.

1) Assess the frequency and appropriateness of prescribing of antipsychotic medication in older adults with BPSD referred to Psychiatry of Old Age service in the West of Ireland (Sligo) by comparing with best practice guidelines.

2) Address identified deficits via quality improvement initiatives within department.

Method. Audit standards were set using draft National Clinical Guidelines and NICE guidelines for prescribing in dementia to develop a study specific audit tool.

Items assessed included: the frequency of review of antipsychotic use, whether or not non-pharmacological methods were trialled, if there was an assessment of benefit of the antipsychotic and discussion or risks, if a reduction/discontinuation of antipsychotic was considered, if metabolic monitoring was achieved.

Clinical records for all patients actively under the care of the clinical team with a diagnosis of BPSD were assessed using this tool at the time of the study.

Result. 49 patients with BPSD were attending the service in this time period. $58 \%(n=29)$ of the entire cohort were prescribed an antipsychotic, most commonly quetiapine. Patients cared for at home showed the lowest levels of antipsychotic use at 50\% $(\mathrm{n}=18)$, while those who were in nursing home $(80 \%, n=8)$ and hospital care $(100 \%, \mathrm{n}=3)$ showed higher rates, though this sample size was too small to demonstrate statistical significance, $\chi^{2}=5.12 \mathrm{p}=0.077$.

Exploration of non pharmacological management of BPSD, documentation of discussion of risks of AP medication (metabolic, cardiovascular, falls, sedation, extrapyramidal), attempt at dose reduction or antipsychotic withdrawal were all achieved in less than $45 \%$ of cases (range $33-45 \%$ ).

Conclusion. This audit revealed higher than expected rates of antipsychotic prescribing in our BPSD cohort. It also revealed suboptimal documentation around the use of antipsychotics in this population during clinical interactions. 
A subsequent intervention to the proforma assessment tool to prompt these discussions improved these behaviours, there was no impact on the rates of antipsychotic prescribing.

Despite increased attention regarding the limited benefits of antipsychotic medication in BPSD their use remains widespread. Due attention must be given to changing this practice in order to protect this vulnerable patient group.

The monitoring and use of Pro re nata (PRN) psychotropic medication for people with learning disabilities on an inpatient ward

\section{Heena Mistry \\ Leeds and York Partnership NHS Trust}

doi: 10.1192/bjo.2021.278

Aims. This project was designed to evaluate the use of PRN medication and PRN monitoring charts on an adult learning disability ward. These charts had been designed by the trust to provide us with a way of monitoring the use of psychotropic PRN medication to ensure monitoring of treatment response, physical health and side effects.

Method. The data were collected from PRN monitoring charts, electronic case notes and electronic prescribing chart records for all patients on an adult learning disability inpatient unit. The sample consisted of 7 patients who had been prescribed and/or received PRN psychotropic medication over a five week period. Quantitative data were derived by simple calculation for the total amount of PRN medication used and number of PRN monitoring charts completed. Qualitative data were collected of prescription charts and PRN protocols which is supposed to guide treatment.

Result. Out of all the incidences where PRN medication was administered, only $64 \%$ of monitoring charts were completed. Out of the 7 patients on the ward, 6 had PRN protocol charts and for only 5 patients these were followed.

Conclusion. Clinical practice must be improved. The results were presented to ward staff and doctors to discuss the implications for patient care and ways to improve clinical practice by ensuring full monitoring of the use of PRN medication to help reduce the overmedication of people with learning disability by improving the use of the PRN charts. NICE guidelines and The Royal College of Psychiatrists have published guidelines on the prescription of psychotropic drugs for people with learning disabilities. NHS England have also published an article to discourage overmedication of people with learning disabilities. There is a risk that doctors are prescribing medication to treat behaviour that is an expression of distress or a mode of communication rather than a mental disorder. Doctors have a responsibility to ensure they have fully assessed the person's potential to benefit from medication before they prescribe. The audit would serve to provide a baseline for this team prior to any audits in the future.

\section{Improving baseline and follow-up physical health monitoring when commencing oral antipsychotics}

Nathan Mitchell ${ }^{1 *}$, Aamer Sajjad ${ }^{2}$, Anna Grocholewska-Mhamdi ${ }^{2}$ and Catherine McMain ${ }^{2}$

${ }^{1}$ Hull York Medical School and ${ }^{2}$ Navigo Heath and Social Care CiC ${ }^{\star}$ Corresponding author.

doi: 10.1192/bjo.2021.279

Aims. NICE guidelines suggest baseline physical health monitoring be performed prior to commencing antipsychotics, in addition to follow-up monitoring for adverse effects for at least 12 months. 'Shared Care Guidelines' were adapted from NICE guidance for local use in North East Lincolnshire. Nevertheless, a local audit published in 2018 reported low compliance with baseline monitoring in community mental health teams (CMHTs) compared to inpatient teams. The parameter most infrequently performed overall was the Glasgow Antipsychotic Side Effect Scale (GASS) questionnaire.

This study aimed to assess whether compliance with baseline physical health monitoring had improved in line with the previous audit's recommendations. Additionally, it aimed to expand on previous findings by adding compliance data for follow-up physical health checks and produce further recommendations to optimise performance.

Method. A retrospective re-audit was performed in NAViGO Health and Social Care to assess compliance with the guidelines for physical health monitoring when commencing antipsychotics in previously antipsychotic-naïve patients. Patient records were examined for which recommended physical health checks were performed at baseline, and at 1-, 3- and 6- months from commencing antipsychotics.

Result. 15 eligible patients were identified to have been commenced on antipsychotics, 8 patients under a CMHT and 7 under an inpatient team. The average overall compliance at baseline for checking 16 parameters was $50 \%$. For the CMHT, compliance was $60 \%$, compared to $38 \%$ for the inpatient team. Across both teams, baseline compliance was highest for renal function tests, liver function tests, and blood pressure and pulse (80\%). For 1-, 3-, and 6- month checks, overall compliance for checking recommended parameters were $33 \%, 29 \%$ and $29 \%$ respectively. GASS monitoring compliance was $7 \%$ at baseline, $0 \%$ at 1 - and 3 -months, $7 \%$ at 6 -months.

Conclusion. The CMHT performed better than the inpatient team at baseline monitoring. This may reflect action on the previous audit's recommendations to increase provision of community 'Wellbeing Health Improvement Service' (WHISe) clinics. However, performance of the GASS questionnaire at baseline was consistent with the previous audit, with similar performance at follow-up extending these findings.

In response, the first recommendation is for Quality Improvement Activities to help improve compliance with the 'Shared Care Guidelines'. This may include CQUINs and further provision of community clinics to improve compliance with both baseline and follow-up checks. Secondly, it is proposed that GASS questionnaires be sent to patients prior to appointments to be completed in advance to avoid further risk of GASS being incomplete.

\section{Audit of inpatient smoking cessation advice}

Flensham Mohamed ${ }^{1 *}$ and Mohamed Bader ${ }^{2}$

${ }^{1}$ Aneuran Bevan University Health Board and ${ }^{2}$ Aneurin Bevan University Healthboard

${ }^{*}$ Corresponding author.

doi: 10.1192/bjo.2021.280

Aims. Audit carried out to assess whether or not patients had been asked about their smoking status during admission onto an acute adult mental health ward, as well as if they had received any smoking cessation advice or offered nicotine replacement therapy.

Background.

- Physical health outcomes in patients with serious mental illness (SMI) are consisitently worse than the general public This is due to multiple factors; adverse effects of medication (including metabolic syndromes with psychotropics) as well as poor lifestyle factors such as smoking status 